\title{
EchoGéo
}

$44 \mid 2018$

Les valorisations territoriales et touristiques du street art

\section{Tour Paris 13 : le cinéma documentaire entre mémoire et scénographie de l'éphémère}

Thomas Lallier

(2) OpenEdition

Journals

Electronic version

URL: https://journals.openedition.org/echogeo/15456

DOI: 10.4000/echogeo.15456

ISSN: 1963-1197

Publisher

Pôle de recherche pour l'organisation et la diffusion de l'information géographique (CNRS UMR 8586)

Electronic reference

Thomas Lallier, "Tour Paris 13 : le cinéma documentaire entre mémoire et scénographie de

l'éphémère", EchoGéo [Online], 44 | 2018, Online since 31 July 2018, connection on 31 July 2021. URL

http://journals.openedition.org/echogeo/15456 ; DOl: https://doi.org/10.4000/echogeo.15456

This text was automatically generated on 31 July 2021.

EchoGéo est mis à disposition selon les termes de la licence Creative Commons Attribution - Pas d'Utilisation Commerciale - Pas de Modification 4.0 International (CC BY-NC-ND) 


\title{
Tour Paris 13 : le cinéma documentaire entre mémoire et scénographie de l'éphémère
}

\author{
Thomas Lallier
}

1 Le $1^{\mathrm{er}}$ octobre 2013, un banal immeuble des années 1950 appartenant à un ensemble de trois unités d'habitation, situé entre la gare d'Austerlitz et le métro Quai de la Gare dans le treizième arrondissement de Paris, allait accueillir un mois durant plus de 25000 visiteurs, certains patientant parfois plus d'une dizaine d'heures avant de pouvoir rentrer dans le bâtiment. Rebaptisé Tour Paris 13, cet édifice de neuf étages appartenant à ICF $^{1}$ Habitat La Sablière, une des sociétés gérant le patrimoine immobilier de la SNCF, devait devenir le lieu de « la plus grande exposition de street art jamais imaginée » selon les propres mots de son concepteur, d'autant plus attractive que le bâtiment et les œuvres produites in situ à l'occasion de l'exposition disparaîtraient avec la démolition du bâtiment lui-même. À l'initiative du galeriste parisien Mehdi Ben Cheikh, appuyé par la mairie du $13^{\mathrm{e}}$ arrondissement de Paris, plus d'une centaine d'artistes originaires de dix-huit pays avaient discrètement investi la Tour durant les six mois précédents et travaillé pour certains jusqu'à la veille de l'ouverture à des œuvres destinées à être ensevelies sous les décombres de l'immeuble. Cette exposition hors-norme succédait notamment à un autre projet de résidence d'artistes éphémère, initié quelque temps auparavant par la galeriste Magda Danysz aux Bains-Douches, une ancienne boîte de nuit du centre de Paris. Mais alors que les Bains-Douches était un espace privé réservé à des invités habitués aux mondanités et que le lieu devait être par la suite réhabilité en établissement hôtelier, le projet de la Tour Paris 13 était beaucoup plus ouvert et radical : visites gratuites ouvertes à tout public durant un mois puis démolition totale du bâtiment lui-même.

2 C'est sans doute cette approche grand public et la grande diversité des formes et des expressions artistiques qui ont attiré les visiteurs et la presse française et internationale, la Tour bénéficiant d'une couverture médiatique sans précédent pour ce 
type d'événement. Ainsi, plusieurs centaines d'articles et de reportages ont évoqué la Tour Paris 13 :

- Nannipieri Luisa. "La Tour Paris 13" : le premier musée virtuel du street art. Télérama [En ligne] http://www.telerama.fr/scenes/la-tour-paris-13-le-premier-musee-virtuel-du-streetart,102196.php 16 septembre 2013;

- Landauro Inti. Tour 13: Parisian tower lures street artists. Wall Street Journal [En ligne] https://www.wsj.com/articles/tour-13-parisian-tower-lures-street-artists-1380552526 30 septembre 2013;

- Hack Susan. Forget the Louvre - Tower 13 is Paris' Hottest New Art Museum. Conde Nast Traveller [En ligne] https://www.cntraveler.com/galleries/2013-10-06/tower-13-art-showparis-france 6 octobre 2013.

Un projet transmédia accompagnait l'événement Tour Paris 13, constitué notamment d'un site internet interactif et participatif et d'un documentaire télévisuel, deux facettes de ce projet monumental que j'ai conçues et réalisées. Respectant la chronologie des visites publiques de l'espace réel de la Tour (IRL - In Real Life), le site internet devait être ouvert durant toute la durée de l'exposition puis « fermé » un mois plus tard. Concrètement, le procédé narratif choisi pour signifier cette fermeture était que le site devait perdre ses couleurs en devenant monochrome (en passant en noir et blanc), à moins que les internautes n'agissent en cliquant sur les 500000 pixels du site pour réactiver les couleurs du site, et donc des œuvres. Pour ce faire ils disposaient de dix jours, mais trois suffirent au vu de l'engouement des internautes. La conception technique du site permettait en outre d'inclure sur la page d'accueil les photographies prises par les visiteurs de la Tour postées sur les réseaux sociaux grâce au hashtag \#tourparis13 et invitait donc les visiteurs à être partie prenante de la diffusion de l'événement, tout en mettant leurs propres images à l'honneur. Lors de la démolition du bâtiment, un dispositif de slow TV a retransmis durant trois jours sur le site la disparition en temps réel de la Tour Paris 13. Ensuite, deux versions du documentaire relatant l'histoire du projet ont été finalement réalisées, la première La Tour Paris 13, de l'art à la poussière, d'une durée de 52 minutes, diffusée sur la chaîne France ô en 2015 (produite par La Blogothèque), et la seconde, Tour Paris 13/Paris Tower 13, d'une durée de 75 minutes, finalisée début 2016 (produite par La Blogothèque, Mirage Illimité et Lester Bernard), destinée aux festivals de cinéma et à la distribution en VOD (Video On Demand). Le site comme le film ont dans leurs domaines respectifs obtenu des distinctions en France et à l'étranger: FWA meilleur site mobile et Design Licks en décembre 2013 pour le site internet; Meilleur Film au Cabo Verde Film Festival - Sal, Cap Vert en 2016 et Best documentary in contemporary art, intallation art, protest art au Master of Art Film Festival - Sofia, Bulgarie en 2017.

Dès les premières rencontres avec les artistes, à partir d'avril 2013, la question s'est posée de savoir ce qu'il était opportun de montrer ou d'éluder, dans la perspective de créer une structure linéaire narrative intelligible pour le spectateur, de faire émerger des personnages et de les inscrire dans un décor. Il faut souligner ici que personne n'imaginait à ce stade l'ampleur du succès de l'exposition, il a donc été décidé de s'en tenir aux éléments scénaristiques tangibles : des artistes (mais sans qu'ils soient encore clairement identifiés ainsi que la nature de leur travail); un lieu avec ses caractéristiques architecturales; et un moment dramatique qui illustrait la fin d'une époque, avec la démolition du bâtiment dans un quartier en mutation. Au tournage, les contraintes de production ne permettaient pas d'assister en permanence aux 
interventions des artistes, mais au-delà de l'acte de prise de vue documentaire, le film devait surtout raconter ce qui motivait profondément ces artistes " urbains ", souvent issus du graffiti, à créer consciemment des œuvres dont ils savaient qu'elles seraient détruites in fine. Ce questionnement a sous-tendu les entretiens avec les artistes et transparait dans le film.

5 Cette approche était pertinente au tournage et déterminante au montage, lorsqu'il a fallu sélectionner qui figurerait dans le film ou pas, et comment donner sa place au bâtiment, au quartier et à la ville de Paris elle-même, plutôt que relater à quel point l'exposition était une réussite, ce qui n'était tout simplement pas le sujet. À l'étape du montage, qui est un moment introspectif où l'on reconsidère chaque plan, chaque séquence à l'aune de l'ensemble du matériau filmique (la totalité des rushes), il s'agissait de construire le film avec l'idée que les œuvres des street artists sont le produit de la ville et de la mémoire. Ces artistes procèdent en effet à une appropriation créative éphémère de l'espace urbain, qui est susceptible de disparaître dans l'heure ou de résister à l'épreuve du temps. Par là même, ils peuvent participer à la constitution d'un corpus via les traces qu'ils laissent dans l'espace réel ou via les photographies et les vidéos de leurs œuvres partagées sur internet, repoussant ainsi l'oubli de l'événement et déployant un terrain de jeu ancré géographiquement à travers d'autres modalités : la singularité du lieu réel, unique (un mur, du mobilier urbain), étant en quelque sorte démultipliée sur les sites reprenant les photographies, et ainsi à travers le monde. En choisissant plusieurs images tirées de la version longue du film documentaire Tour Paris 13/Paris Tower 13, nous proposons d'évoquer le thème de la mémoire des lieux telle qu'elle apparait implicitement ou explicitement à travers les installations in situ des artistes.

6 La Tour Paris 13, située sur le quai d'Austerlitz entre la rue de Bellièvre et la rue Fulton dans le $13^{\mathrm{e}}$ arrondissement de Paris, appartenait à un groupe de trois immeubles datant d'après-guerre, au sein d'un quartier destiné à être démoli. En effet, initiée au début des années 1990, la Zone d'Aménagement Concertée de Paris Rive Gauche, pilotée par la société d'économie mixte SEMAPA, avait pour mission de restructurer une vaste étendue essentiellement composée de bâtiments techniques et industriels, située entre la Gare d'Austerlitz et la Porte d'Ivry, délimitée par les voies de chemin de fer et la Seine. Le galeriste Mehdi Ben Cheikh, qui cherchait à l'époque des grands murs aveugles pour son projet de musée de street art à ciel ouvert, était habitué à dialoguer avec les bailleurs sociaux gérant les grands ensembles immobiliers du $13^{e}$, la galerie Itinerrance étant elle-même basée dans cet arrondissement. Lorsque s'est présentée la possibilité de peindre les façades de l'immeuble (illustration 1) et qu'il s'est avéré que cet immeuble lui-même serait détruit prochainement (ce qui n'est pas le cas des autres projets de fresques initiés par Mehdi Ben Cheikh), le galeriste a proposé d'investir également l'intérieur du bâtiment. Le prix du foncier parisien ayant fortement évolué à la suite du réaménagement de la zone et l'immeuble ne répondant plus aux normes d'habitation contemporaines, la démolition était inéluctable. 
Illustration 1 - L'îlot Fulton

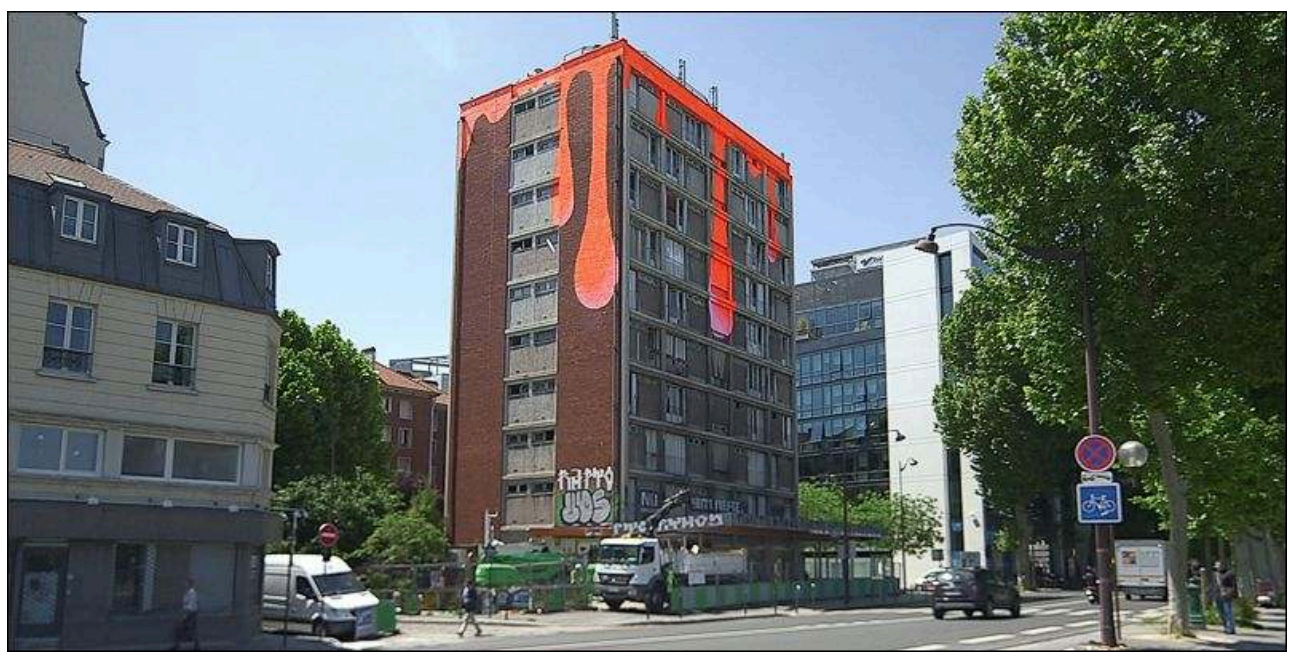

Auteur : Th. Lallier

7 Mehdi Ben Cheikh a proposé à l'artiste El Seed de peindre cette façade, devenue emblématique du projet. Le calligraffiti d'El Seed, blanc aux contours noirs sur fond orange fluorescent est une interprétation personnelle d'un vers de Charles Baudelaire «La forme d'une ville change plus vite, hélas, que le cœur d'un mortel $»^{2}$ en référence au sort de la Tour elle-même et partant, à toute cette partie du $13^{\mathrm{e}^{\circ}}$ arrondissement entre la Gare d'Austerlitz et la porte d'Ivry.

Dans une séquence du film (illustration 2), El Seed fait remarquer que dans le contexte actuel, chacune de ses tentatives de peindre une fresque en arabe a été refusée par la municipalité. Il évoque une possible "arabo-scriptophobie» des édiles qui ne voudraient pas affronter la vindicte des habitants, associant a priori la graphie arabe à des textes religieux, inacceptables dans le contexte laïc français. C'est sans doute grâce au caractère éphémère de la Tour Paris 13 qu'El Seed a pu enfin réaliser un graffiti en langue arabe aux dimensions monumentales.

Illustration 2 - El Seed sur sa nacelle

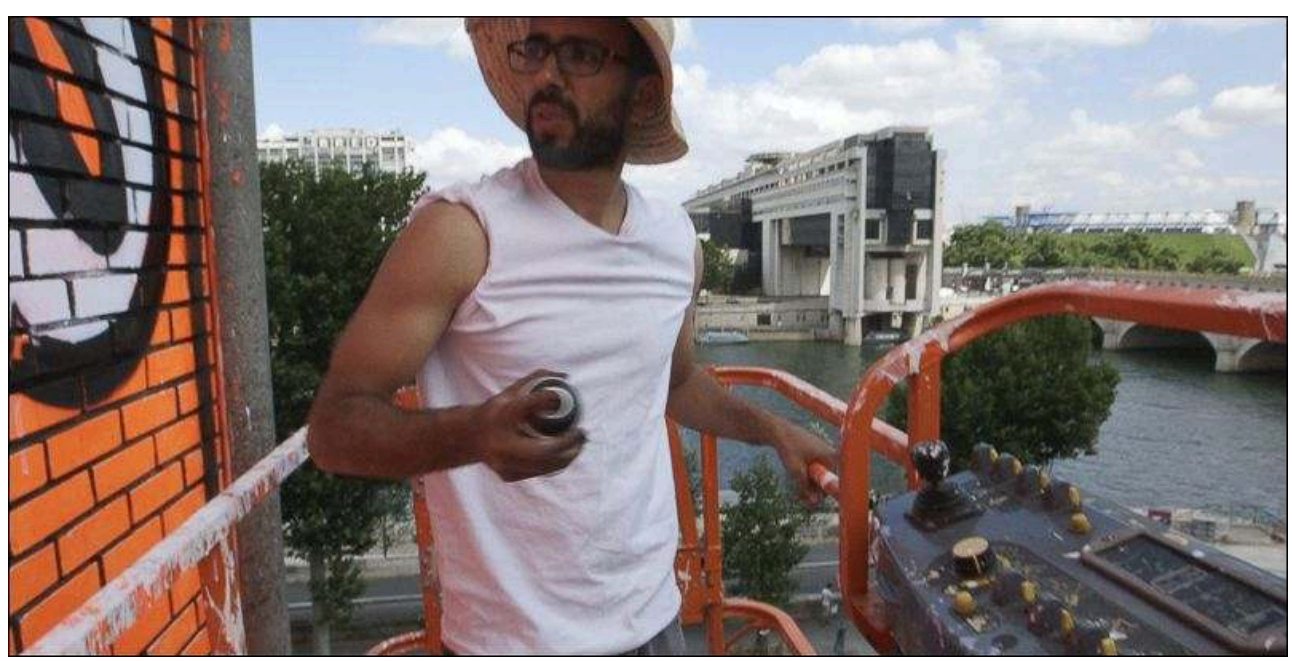

Auteur: Th. Lallier. 
$\mathrm{Au}$ moment où furent construits les immeubles de l'îlot Fulton (visible à droite de l'illustration 3), les quartiers situés de part et d'autre de la Seine, entre les gares de Lyon et d'Austerlitz et les portes de Bercy et d'Ivry, étaient essentiellement des zones dédiées à l'approvisionnement de la population parisienne, notamment en vins et spiritueux rive droite, en farines et matériaux de construction rive gauche. La rive droite a été réhabilitée au début des années 1980. La réhabilitation de la rive gauche est aujourd'hui en voie de finalisation, l'îlot Fulton étant un des derniers témoins de cette immense zone industrielle du « Vieux Paris ».

Illustration 3 - Vue depuis le $7^{\mathrm{e}}$ étage de la Tour

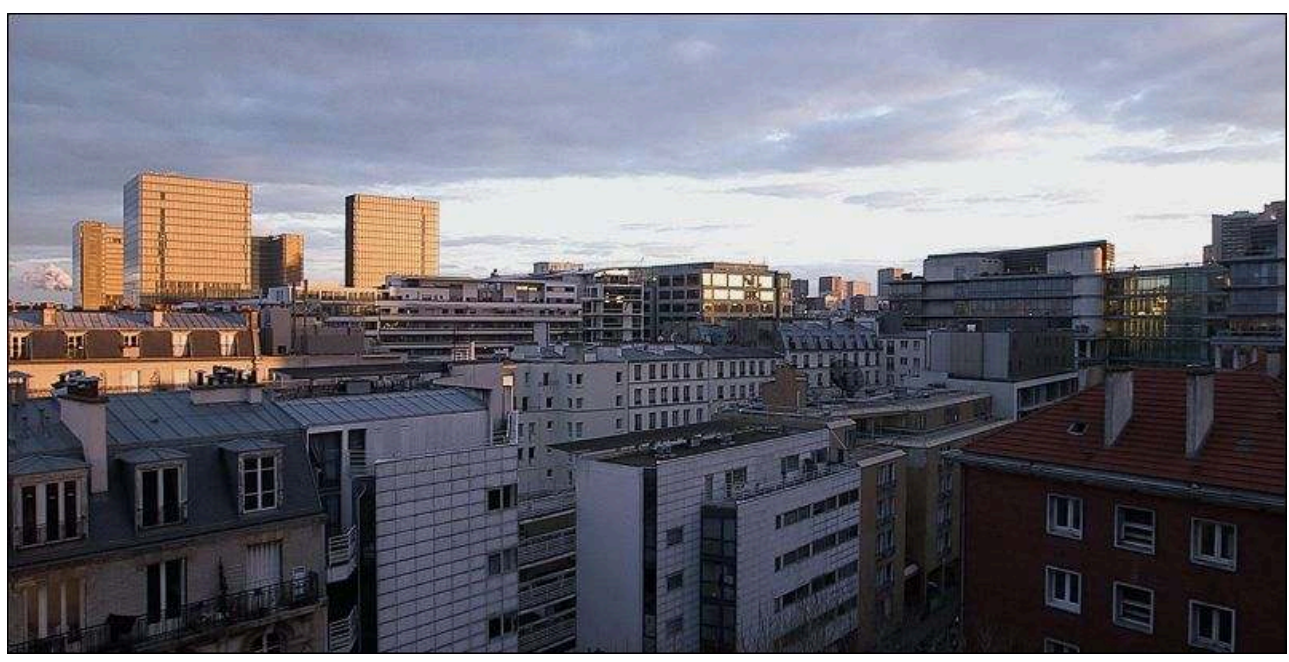

Au commencement du tournage, il était envisagé d'évoquer l'environnement immédiat de la Tour en termes urbanistiques et architecturaux, peut-être parler de la Grande Bibliothèque, peut-être parler des «Frigos ", des entrepôts frigorifiques désaffectés épargnés par les opérations de réaménagement et haut lieu du graffiti dans les années 1990. Mais, finalement, le choix fut d'être au plus près des artistes, des œuvres auxquelles ils travaillaient dans la Tour et plus largement d'être attentif aux motivations profondes de leur geste artistique : pourquoi ils faisaient ce qu'ils faisaient et pourquoi ils avaient choisi d'intervenir dans l'espace urbain, plutôt que dans un atelier par exemple. En termes de dramaturgie, le film s'efforce d'amener le spectateur à accepter que ces œuvres, aussi fortes, expressives, émouvantes qu'elles soient, seraient irrémédiablement détruites. Ainsi la disparition du bâtiment devait aussi incarner la disparition des œuvres, illustrant la fragilité et les limites des productions humaines, qu'il s'agisse de construction ou de travail artistique. Dans cette même image finale, au-delà de la structure dramaturgique du récit filmique, il s'agit aussi d'évoquer l'essence de l'art urbain, qui a besoin de surfaces, de supports, toujours renouvelés. Si la destruction d'un bâtiment des années 1950 est vraisemblablement nécessaire pour accompagner une phase nouvelle de développement urbain, la disparition des œuvres d'art urbain qu'il renferme permettra sans doute à de nouvelles productions de voir le jour, sous d'autres formes, d'autres modalités. À ce titre, le graffiti parisien, comme les murs et les bâtiments qui lui ont servi de support dans les années 1980 par exemple, n'a pas été patrimonialisé. Pour autant, l'art urbain n'a pas disparu à Paris et continue de se développer. Le graffiti des années 1980 subsiste encore dans les mémoires notamment grâce à la photographie. 
Illustration 4 - Katre

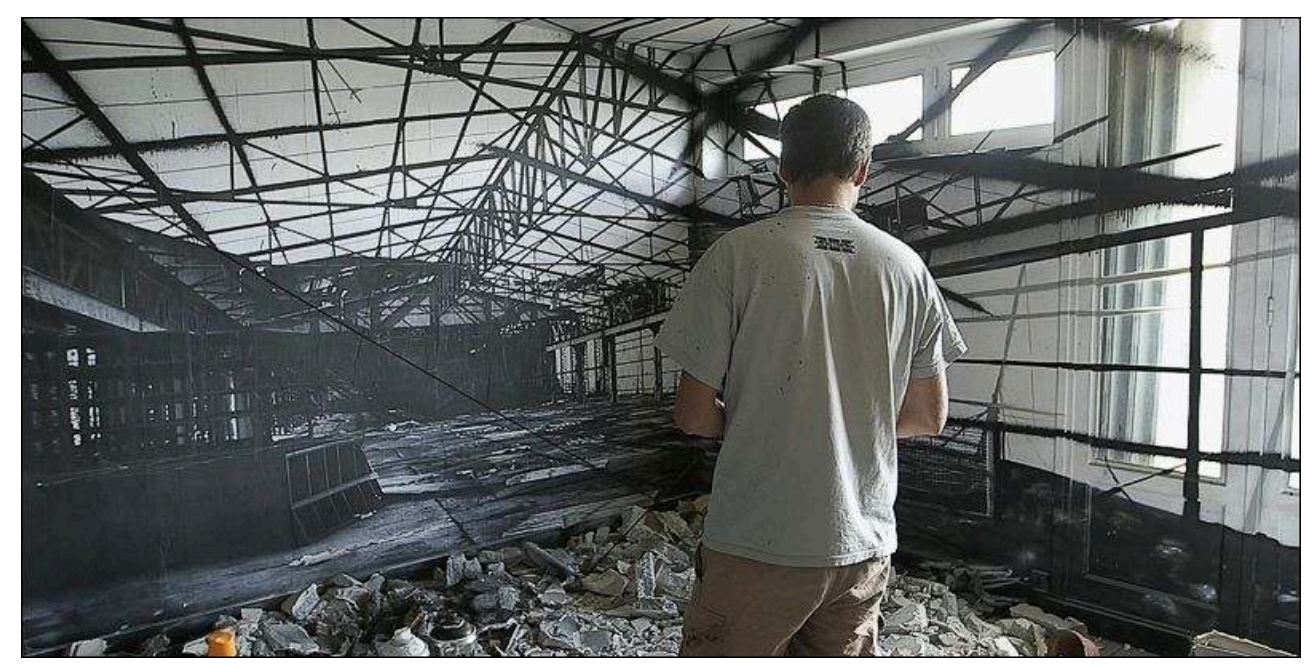

Auteur : Th. Lallier.

11 L'installation de l'artiste Katre dans la Tour est très intéressante, car Katre crée une sorte de jeu de miroirs entre les espaces et les temporalités. Cet artiste pratique l'exploration urbaine (UrbEx), il réalise des photographies de friches industrielles et autres endroits à l'abandon et exploite visuellement ce matériau en lui donnant une seconde vie à travers ses œuvres. Ses images de lieux vraisemblablement déjà détruits au moment où il les mettait en scène dans l'appartement qui lui était attribué résonnaient d'autant plus fort que la Tour Paris 13 devait elle-même être détruite à son tour. Katre travaille avec la photographie, qui est par essence un médium de la mémoire, de l'enregistrement de ce qui a été. La séquence avec Katre apparaît comme une sorte de méta-niveau de compréhension des enjeux narratifs du film : au-delà du récit d'un événement artistique hors-norme, ce film documentaire peut aussi être envisagé comme un film sur la mémoire.

$12 \mathrm{Au}$ cours de leurs interventions, certains artistes adeptes de l'UrbEx ont découvert comment accéder à un ensemble de pièces de l'immeuble rendues inaccessibles au public (illustration 5). L'endroit qu'ils ont investi est une station-service désaffectée, qui était située au rez-de-chaussée de la Tour, et condamnée depuis plusieurs années. Avec l'évolution des normes de sécurité et de pollution, l'implantation de ce type d'aménagement en rez-de-chaussée d'habitation est maintenant interdite.

13 L'existence même de ce lieu symbolise là aussi un passé révolu, auquel appartient l'immeuble. Certains des artistes parisiens de la Tour se remémorent qu'ils venaient faire le plein de leurs deux-roues dans cette station-service, dans les années 1990. 
Illustration 5 - La pièce secrète

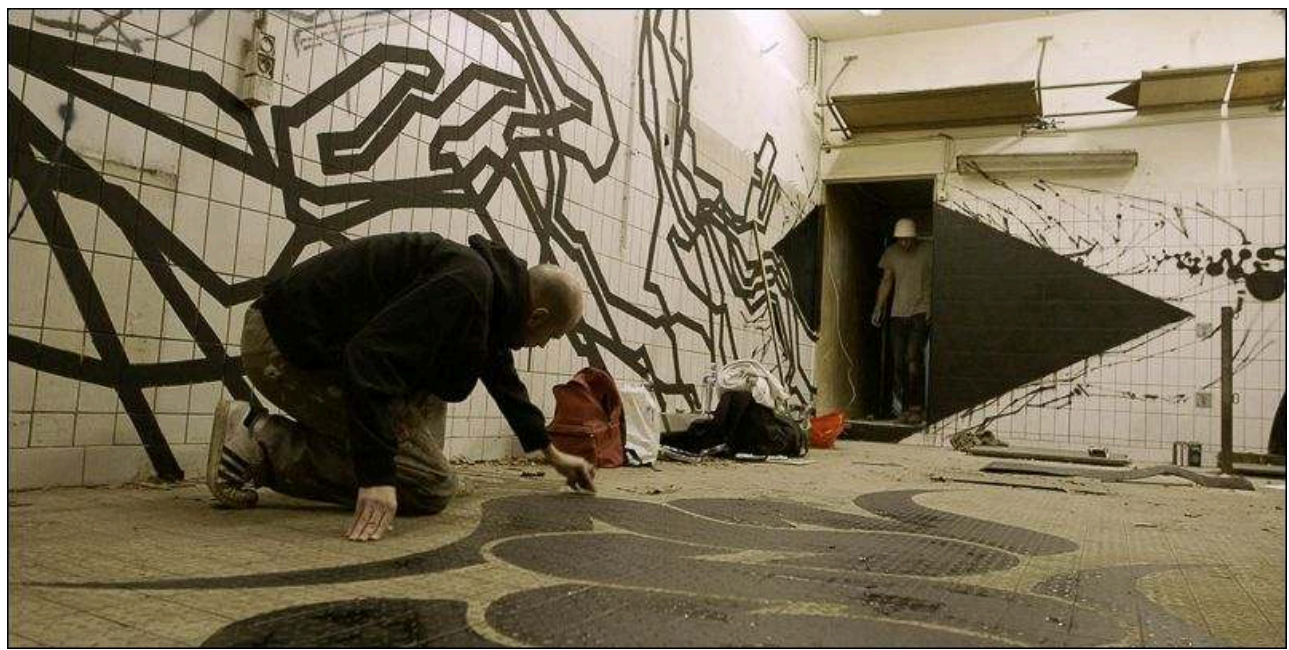

Auteur : Th. Lallier nombreux venant d'horizons très divers, des amateurs de street art certes mais aussi des promeneurs et des curieux motivés par la perspective d'une expérience visuelle originale. À la différence des établissements de type muséal, l'expérience de l'exposition démarrait à l'extérieur du bâtiment avec des œuvres visibles sur les façades et le mobilier urbain, ce qui aidait la foule à patienter, souvent plusieurs heures, en attendant de pénétrer dans la Tour.

Illustration 6 - La Tour Paris 13 lors des visites

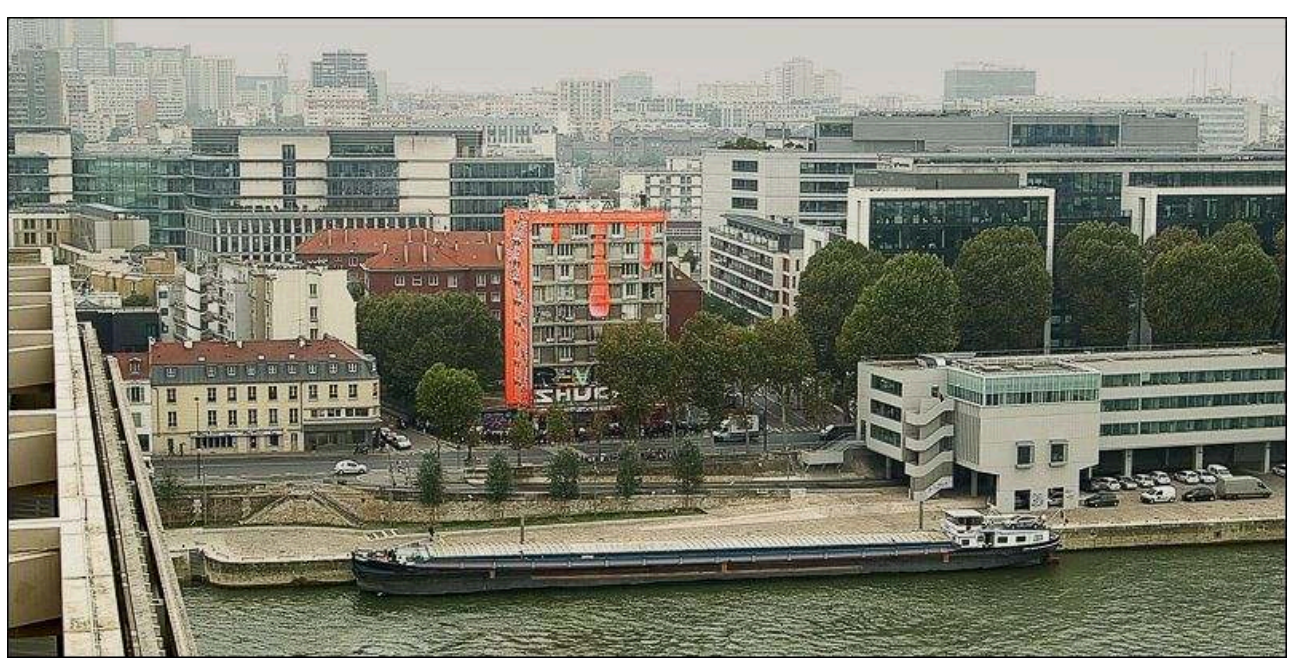

Auteur : Th. Lallier

Cet immeuble à l'architecture banale est devenu, du fait de l'intervention des artistes et de sa disparition programmée, un véritable phénomène, haut lieu culturel et artistique, voire une destination touristique éphémère. L'image (illustration 6) montre à quel point le bâtiment est très majoritairement entouré de constructions récentes et 
comment la Tour Paris 13, toute colorée, détonnait dans le paysage urbain plutôt monotone des immeubles environnants. Cette irruption de la couleur et de formes graphiques diverses rencontra l'adhésion de la plupart des riverains, invités par des associations de quartier à visiter la Tour avant les visites publiques. Elle suscita aussi une certaine hostilité, exprimée (mais peu soutenue) sous forme d'une pétition (sur le site MesOpinions.com) pour que cessent les visites et que la Tour soit détruite dans les plus brefs délais. Une contre-pétition a immédiatement vu le jour sur le même site, demandant au contraire le prolongement des visites, pour que les non-Parisiens puissent aussi voir les œuvres in situ.

17 L'illustration suivante tirée de la séquence de démolition est une forme de mise en abyme : on peut y voir au premier plan la démolition en cours de la Tour et en arrièreplan un immeuble similaire à la Tour mais non investi par des artistes, faisant partie du même groupe d'immeubles et destiné à subir le même sort.

Illustration 7 - Démolition

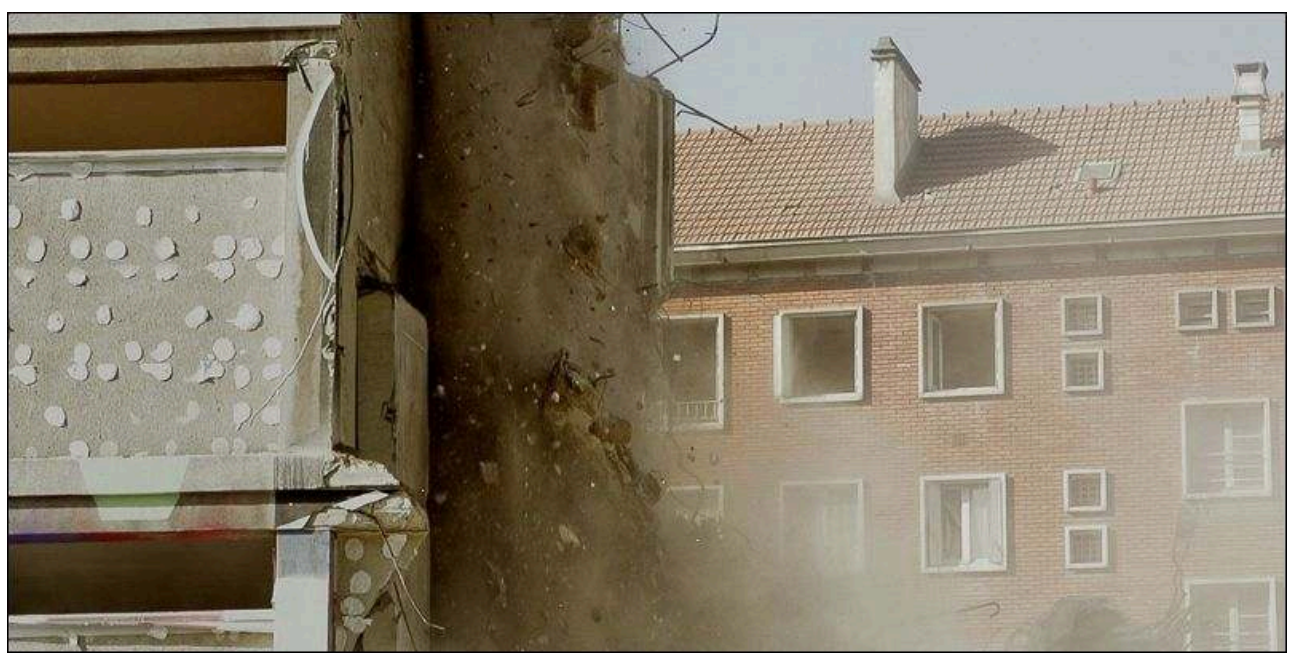

Auteur : Th. Lallier.

18 Les dernières images de la démolition ont été filmées au ralenti, ce qui permet de faire exister la Tour plus longtemps aux yeux des spectateurs. Ce temps suspendu est aussi un temps pour se remémorer les œuvres aperçues dans le film, alors qu'elles sont en train de disparaitre, et convoque ainsi la propre mémoire du spectateur et les images qu'il retiendra du film.

Ce plan large du film (illustration 8) montre le processus de démolition retenu, la technique du " grignotage ", et le contexte topographique de la Tour Paris 13, avec à l'arrière-plan le pont de Bercy, la passerelle Simone de Beauvoir et la bibliothèque François Mitterrand. Le " grignotage » consiste à détruire l'immeuble par l'action d'une pince métallique, de haut en bas en respectant la structure de l'édifice pour éviter qu'il ne s'effondre, ce qui permet notamment de trier les matériaux de construction de manière sélective, pour pouvoir les recycler et les valoriser.

20 Le choix de cette technique de démolition, au ralenti pour ainsi dire (la démolition a duré trois jours), a laissé suffisamment de temps pour pouvoir multiplier les angles de prises de vues. 
Illustration 8 - Démolition plan large

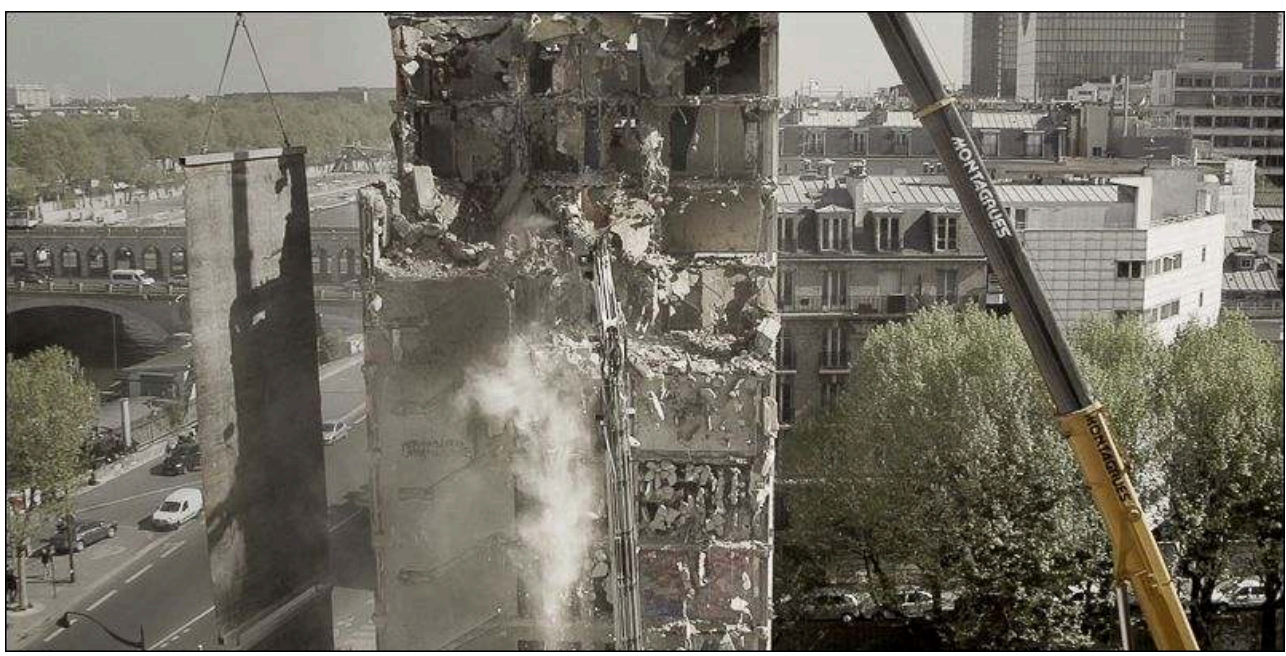

Auteur : Th. Lallier.

21 La version longue du film (75 minutes), qui a été projetée pour la première fois au festival de cinéma de Varsovie (Pologne) en octobre 2016 puis au festival de cinéma de Leeds (Royaume-Uni), a obtenu un prix au festival de films sur l'art de Sofia (Bulgarie) et a également été projetée dans divers festivals au Cap Vert, aux îles Caïman, en Croatie, en Finlande, en Nouvelle-Zélande, au festival de films d'architecture de Lund (Suède), et dans un festival de street art à Grenoble. Les rencontres avec le public organisées à l'issue des projections montrent à quel point la disparition de la Tour Paris 13, à la fin du film, est perçue par les spectateurs comme un événement sensible, émotionnel, investi de la mémoire des images des œuvres.

Pour le réalisateur, qui est aussi un raconteur, c'était le but de ce film : faire en sorte que les spectateurs repartent de la projection avec en tête des moments qu'ils n'ont pas vécus, mais qu'ils se sont intimement appropriés.

\section{BIBLIOGRAPHY}

Baudelaire C., 1861. Les Fleurs du mal. Paris, Poulet-Malassis et de Broise, 319 p.

Deiss J., 2014. Tour Paris 13 : Quand le transmédia grave l'art urbain dans le web. Slideshare https://fr.slideshare.net/Veille_Digitale/tour-paris13, consulté le 25 novembre 2014.

Gracq J., 1985. La Forme d'une ville. Paris, José Corti, 213 p.

Lamin L., 2014. Tour Paris 13 : Le transmédia au service de l'art - Etude de Cas. Circulation, Vol. 4, McGill University, Montréal, Canada et Academia.edu, https://www.academia.edu/9706115/Tour_Paris_13_Le_transm\%C3\%A9dia_au_service_de_lart__Etude_de_Cas, consulté le4 décembre 2014. 
Ramain C., 2013. La Tour Paris 13, exemple de transmedia activism. Socialtv.fr, http:// www.socialtv.fr/dispositifs/transmedia-crossmedia/la-tour-paris-13-exemple-de-transmediaactivism/, consulté le 12 novembre 2013.

Site officiel du film documentaire, http://www.paristower13.com/

Site de la Galerie Itinerrance, http://itinerrance.fr/hors-les-murs/la-tour-paris-13/

\section{NOTES}

1. ICF signifie Immobilière des Chemins de Fer.

2. Citation via l'essai de Julien Gracq (1985), La Forme d'une ville, du poème de Charles Baudelaire, Le Cygne, tiré des Tableaux parisiens des Fleurs du Mal.

\section{AUTHOR}

\section{THOMAS LALLIER}

Thomas Lallier, thlallier@gmail.com, est réalisateur indépendant. Sa filmographie peut être consultée sur http://www.thomaslallier.com/director/ 